J.K. Ghosh, S.K. Mitra, K.R. Parthasarathy and B.L.S. Prakasa Rao (eds.), Statistics and Probability: A Raghu Raj Bahadur Festschrift

Published by Wiley Eastern Limited, Publishers, (1993), 371 - 380

\title{
The Bertrand-Borel Debate and the Origins of the Neyman-Pearson Theory ${ }^{1}$
}

\author{
E.L. LEHMANN \\ University of California, Berkeley
}

\begin{abstract}
The views of the mathematicians Bertrand and Borel on hypothesis testing and the usefulness of probability models are reviewed as background to the Neyman-Pearson theory. There is a discussion of Neyman's claim that these views had an influence on his work with Pearson, and of Pearson's denial of this claim.
\end{abstract}

\section{Introduction}

Throughout the $19^{\text {th }}$ Century, hypothesis testing was carried out in various different areas of application along the lines laid down in the "Théorie Analytique des Probabilités" by Laplace $(1812,1814,1820)$ and in Poisson's "Recherches sur la Probabilité des Jugements" (1837). Nearly all of this work was asymptotic, based on De Moivre's normal approximation to the

\footnotetext{
${ }^{1}$ Research supported by NSF Grant No. DMS-8908670.

AMS Subject Classifications: Primary 62-03; secondary 62 F 04

Key words and Phrases: Hypothesis Testing, Neyman-Pearson Theory, Probability Model.
} 
binomial and Laplace's central limit theorem. Toward the end of the century, the approach was summarized by Edgeworth (1895) and extended in an important new direction by Karl Pearson (1900).

A new phase, small-sample theory, was ushered in by Student (1908) and was developed by Fisher in a series of papers culminating in his book "Statistical Methods for Research Workers" (1925). In all the work up to this point the test was taken as given (on intuitive grounds) and the emphasis was on the calculation of the $p$-value or, when a Bayesian approach was used, on the probability of the hypothesis being true.

The final step, asking how to choose the test, was taken by Neyman and E.S. Pearson $(1928,1933)$ who formulated the problem and to a large extent solved it. These authors give somewhat different accounts of the main influences that inspired them in this work. Pearson $(1939,1966)$ attributes the suggestion that the appropriate test must depend on the alternatives against which the hypothesis is to be tested to a letter of Student while Neyman cites the influence of a debate between Bertrand and Borel.

The Bertrand-Borel debate ${ }^{2}$ is concerned not only with the problem of how to choose a test, but also with the more fundamental question of whether calculations of $p$-values are appropriate at all since they are based on probability models that at best are rough approximations of a much more complex real situation. In presenting the views of Bertrand and Borel in sections 2 and 3 , we shall be concerned with both these aspects of the debate. Sections 4 and 5 discuss its relation to the Neyman-Pearson theory.

\section{Bertrand's views}

Neyman's source for Bertrand's views was Bertrand's book "Calcul des Probabilités (1889, 1897, 1907). The author explains the purpose of his book in a short preface. Probability theory is one of the most attractive branches of the mathematical sciences, he says, and yet one of the most neglected. He suggests that one of the reasons for this state of affairs may be Laplace's book (the Théorie Analytique), which is indispensable for the study of probability theory, but requires a great deal of mathematical preparation. Bertrand's book is to remedy this situation by providing an account of the most useful and best known results with the simplest possible proofs.

Emulating Laplace, Bertrand begins his book with a nontechnical essay, "Les lois du hasard" [The laws of chance], which gives an introductory overview. ${ }^{3}$ Besides the essay, the relevant chapter for the present purpose is chapter 7 on the probability of causes. Much of this chapter is devoted

\footnotetext{
${ }^{2}$ This seems the best way to describe the situation although it was not a debate in the sense of a formal discussion. Bertrand stated certain views in his probability text of 1889 . He was no longer alive when Borel criticized them rather sharply in a popular book on probsbility in 1914 .

${ }^{3}$ However, in contrast to Laplace's ebullience and optimism, Bertrand's attitude toward applications is rather negative.
} 
to an attack on hypothesis testing on the grounds that the plausibility of a hypothesis depends on the prior probability of it being true, which is rarely known.

Then, speaking about Michell's famous problem ${ }^{4}$ of whether the closeness to each other of the six (visible to the naked eye) stars forming the Pleiades can be attributed to chance, he changes the direction of attack. He again first refers to the unavailability of prior probabilities, but then asks: "How can we decide on the unusual results that chance is incapable of producing?" and continues (p.166)

"The Pleiades appear closer to each other than one would naturally expect. This statement deserves thinking about; but when one wants to translate the phenomenon into numbers, the necessary ingredients are lacking. In order to make the vague idea of closeness more precise, should we look for the smallest circle that contains the group? the largest of the angular distances? the sum of squares of all the distances? the area of the spherical polygon of which some of the stars are the vertices and which contains the others in its interior? Each of these quantities is smaller for the group of the Pleiades than seems plausible. Which of them should provide the measure of implausibility? If three of the stars form an equilateral triangle, do we have to add this circumstance, which is certainly very unlikely apriori, to those that point tc a cause?"

What Bertrand seems to consider here is the choice between a number of possible test statistics, small values of which are significant. However, in the last sentence he addresses an additional issue, namely what features of the available material to include in the observations to be analysed.

Beyond these particular questions, Bertrand here also raises doubts about the scientific validity of such a probabiiistic approach to testing. In the introductory essay he goes much further. After discussing Michell's own analysis, he states ( $p . x v i i i)$ : "The application of such calculations to questions of this kind is a delusion and an abuse."

Quite generally, Bertrand opposes probability models, particularly in the social sciences, as quite unrealistic ${ }^{6}$ and this aspect of the debate continues today. His statement concerning Laplace's law of succession: "If time is to confirm and increase [the conviction that the sun will rise tomorrow], it will be by the discovery of the laws of astronomy and not by the repeated success of ar analogous game of chance", is echoed in recent writing of David Freedman (for example, Freedman (1985) and (1987)).

\footnotetext{
4 For recent reviews of some of the extensive literature on this problem see Gower (1982) and Dale (1991).

${ }^{5}$ Since copies of the book by Bertrand (and that of Borel) cited here are easily available, I have translated the passages to be quoted for easier reading. The page references are to the edition of 1907 .

${ }^{6}$ For further discussion of his views see for example Stigler (1986) and Daston (1988).
} 


\section{Borel's reply}

A very different position is taken by Borel in his book "Le Hasard" (1914); the passages given here in translation are from the revised edition of (1948). His treatment of these issues occurs in chapter 4 which, like Bertrand's corresponding chapter, is entitled "probability of causes". After discussing Bayes' theorem, Borel mentions the example of the frequency of male births and restates it as an urn problem. After some discussion he points out that "this example shows the particular form that problems of causes often take on. This form is the following: Is such and such a result due to chance or does it have a cause? It has often been observed how much this statement lacks in precision. Bertrand has strongly emphasized this point. ${ }^{7}$ But whatever objection one can raise from a logical point of view cannot prevent the preceding question from arising in many situations: the theory of probability cannot refuse to examine it and to give an answer; the precision of the response will naturally be limited by the lack of precision in the question; but to refuse to answer under the pretext that the answer cannot be absolutely precise, is to place oneself on purely abstract grounds and to misunderstand the essential nature of the application of mathematics. True, the calculations provide an exact answer to every exact question; but in practice the questions are never exact: the given experimental facts necessarily permit a certain amount of play; the same lack of precision then affects the result of the calculations and the would-be absolute theoretical precision of these calculations is pure delusion."

Borel next turns to problems in astronomy and after some introductory remarks quotes the passage about the Pleiades from Bertrand that was given in the preceding section. Referring to Bertrand's equilateral triangle, he writes: "If one has observed a number such as that quoted above [1.342517] as evaluation of an angle in tenths of seconds (in the decimal system), one would not think of asking to know the probability that this angle is exactly $13^{G} 42^{\prime} 51^{\prime \prime} 7$; because one would never have asked that precise question before having measured the angle...

"The question is whether one has the same reservations in the case in which one states that one of the angles of the triangle formed by three stars has "une valeur remarquable" [a striking or noteworthy value], and is for example equal to the angle of the equilateral triangle $\left(66^{G} 66^{\prime} 66^{\prime \prime}\right){ }^{8}$ or to a half right angle: 50 decimal degrees, or 5.000 .000 tenths of a second. Here is what one can say on this subject:

"One should carefully guard against the tendency to consider as striking an event that one has not specified beforehand, because the number of

\footnotetext{
${ }^{7}$ Borel here refers to Bertrand's discussion of the different interpretations that can be given to the idea of randomness, as illustrated by his well-known treatment of Buffon's needle problem; cf Bertrand (1889, ch.1).

${ }^{8}$ The old-fashioned french angular measure "grade" is a decimal measure; a right angle is 100 grades.
} 
such events that may appear striking, from different points of view, is very substantial.

"In contrast to this, the question of grouping [i.e. clustering] in space is one that it is natural to ask apriori; the theory of probability does not enable one to resolve it with certainty but makes precise the conclusions one can draw from observations.

"This precision is quite useful, because it often effectively replaces inferences at which one arrives too quickly by 'common sense' ... Between the two extreme situations (those in which the results clearly are or are not significant there are many others, namely those in which the probability lies between for example one hundredth and one hundred-thousandth: the value [the probability] has between these limits is not at all useless and what one can learn from it may be very valuable, no matter what Bertrand thinks".

In summary, Bertrand raises two questions: (i) on what grounds to prefer one test statistic to another; (ii) whether the calculation of a $p$ value, once the test statistic has been chosen, is at all useful in view of the shaky assumptions underlying the typical probability model.

To the first point Borel replies with the idea that the test statistic should represent some striking feature of the situation; on the second he asserts his unshaken faith in the value of probabilistic calculations even though they are only approximations to the true values.

It should be noted that neither of these authors offers any direct suggestion that the test should be based on more plausible alternatives although both Paul Lévy and Neyman were later to interpret Borel's comments in this way.

\section{Circonstances remarquables}

The first mention of the views of Bertrand and Borel in Neyman's papers occur in Neyman (1929) where he refers to Bertrand's critique of the Bayesian approach to hypothesis testing and then mentions: ${ }^{9}$

"M. Borel who also discusses analogous questions, observes that the correct judgment concerning a statistical hypothesis depends on the choice of the aspect of the observed fact $f(E)$ which in some way must be 'remarquable'. This idea of a remarkable aspect which is a bit vague can be made precise if one agrees to consider also alternative hypotheses."

The debate between the two french mathematicians is discussed at greater length in Neyman and Pearson (1933), and Neyman returns to it again in Neyman (1935) where he writes: ${ }^{9}$

"... the origin of the general theory of testing hypotheses is linked to most interesting remarks of french mathematicians, particularly of $J$.

\footnotetext{
${ }^{9} \mathrm{My}$ translation of the original french.
} 
Bertrand (1889) and M.E. Borel (1932). They considered the process of hypothesis testing which is basically the one employed by the english school...

"It is known that Bertrand was skeptical about the scientific value of the results of such a process. On the other hand, $M$. Borel insisted that such a result can be valid provided the feature $f(E)$ of the observed facts that serve as basis for testing $N$, be 'en quelque sorte remarquable'.

"This last remark preceded a series of works by Neyman Pearson, and others] which basically strove to give a precise meaning to Borel's somewhat vague words..."

How much importance Neyman attached to the influence of Borel's remark can be seen from the fact that late in life he returned to it again in Neyman (1977), an account of his views on statistics. He mentions that the methodology for testing hypotheses "was the _subject of a lively discussion by Borel and others", and goes on to say that "Borel was optimistic but insisted that: (a) the criterion to test a hypothesis ... must be selected not after the examination of the results of observation, but before, and (b) this criterion should be a function of the observations 'en quelque sorte remarquable'. It is these remarks of Borel that served as an inspiration to Egon $\mathrm{S}$. Pearson and myself in our effort to build a frequentist theory of testing hypotheses".

Neyman sent a preprint of this paper to E.S. Pearson who expressed himself" delighted with it ("so delighted that I have started devouring it, although only received five hours ago!" he wrote) and who attached to his reply to Neyman a large number of comments. In one of the first of these he takes exception to Neyman's attribution of inspiration cited above. He writes:

"I remember that you produced this quotation when we began to get our [1933] paper into shape and write it up. This must have been during the period 1930/31, or even as early as my summer visit to Poland of 1929. I had not heard of the Borel quotation until you started to draft the introductory section of our paper. The words may have for a year or two been acting as an inspiration to you, but while I at once felt the words relevant and important, my approach was a different one, and was not I think altered by inspiration from the words."

Pearson goes on to say that in 1925 or 1926 he had begun to question the basis of the $t$-test and to ask: Why use that particular statistic? and that this led to the question: 'How choose the rejection region?' He then continues:

"The above stages led up to Borel's requirement of finding ... a criterion which was 'a function of the observations 'en quelque sorte remarquable'.

"Now my point is that you and I (perbaps my first leading) had our-

\footnotetext{
${ }^{10}$ In an unpublished letter of September 28,1976 , which is contained in the Neyman Collection of the Bancroft Library, University of California, Berkeley.
} 
selves reached the Borel requirement independently of Borel, because we were serious humane thinkers; Borel's expression neatly capped our own requirement."

As mentioned in the introduction, a strong outside influence had also affected Pearson's thinking. This was a letter ${ }^{11}$ by Student written in an answer to a question by Pearson. This letter contains the crucial suggestion: "If there is an alternative hypothesis which will explain the occurrence of the sample with a more reasonable probability ... you will be very much more inclined to consider that the original hypothesis is not true."

Pearson must very soon have communicated this idea to Neyman because a letter of Neyman's to Pearson, dated December 9, 1926 begins: "I think that to have the possibility of testing it is necessary to adopt such a principle as Student's, ..." (Reid (1982), p.70). On the other hand, there appears to be no mention of Borel in this or other letters of Neyman's to Pearson of this period. This combination of circumstances lends some support to Pearson's recollections. ${ }^{12}$

It is interesting to note that Neyman was not the only one on whom Borel's discussion of tests of randomness made a deep impression. Paul Lévy in his "Calcul des Probabilités (1925) treats this topic in a section which he acknowledges to be largely borrowed from Borel. He there also mentions the role played by "propriétés remarquables" and goes on to analyse the meaning of this phrase. And he comes to the conclusion which - by whatever common or separate routes - Neyman and Pearson were also to reach. "Une propriété remarquable", he writes, "est une propriété facile à expliquer par une cause autre que le hasard." 13 (p.93). [A striking property is a property that is easy to explain by a cause other than pure chance.]

\section{The Bertrand-Borel debate and the Neyman-Pearson theory}

The basic concepts of the Neyman-Pearson theory: rejection region, type 1 error, alternatives to the hypothesis, and power, have became so embedded in our statistical language and thought that it is difficult for us to see the situation as it was in the first quarter of the century. The debate of the French probabilists of that period provides a window into their thinking.

What strikes the modern reader is the vagueness of the discussion which is of course inevitable when no conceptual framework and suitable terminology are available. As a partial explanation of "the obscurity which envelops the theoretical bases of statistical methods" Fisher in his fundamental paper, "On the mathematical foundations of theoretical statistics" (1922) suggests that "it appears to be widely thought, or rather felt, that in

\footnotetext{
${ }^{11}$ This letter of May 11, 1926 was published in part in Pearson (1939).

12 The Neyman-Pearson story is told from Pearson's point of view in Pearson (1966) and from Neyman's in Reid (1982).

${ }^{13}$ Neyman's only reference to Lévy in this general context is in Neyman (1932) where be attributes the concept of "remarquable" characters to "E. Borel and P. Lévy" in the books quoted here.
} 
a subject in which all results are liable to greater or smaller errors, precise definition of ideas or concepts is, if not impossible, at least not a practical necessity."

In this paper Fisher did in fact create the needed theoretical framework in the context of estimation. He formulated his theory in terms of a parametric family of distributions (of the observations), posed the problem of choosing a suitable estimator, defined a measure of accuracy, and introduced both relative and absolute efficiency. However, Fisher did not extend the theory to hypothesis testing, which - like his predecessors he discussed only in the context of specific examples or classes of examples.

It remained for Neyman and Pearson to formulate a general theory of hypothesis testing. Fisher's parametric families provided a way to specify both hypothesis and alternatives. Instead of speaking, as Bertrand and Borel had done, of choosing among vaguely indicated test criteria, the class of available tests is now clearly defined as the class of all possible level $\alpha$ rejection regions. Furthermore the concept of power made possible a comparison of the advantages and disadvantages of different tests and led to maximal power as a principle for chosing a "best" test.

There is a world of difference between the vague musings of Bertrand and Borel, and the clearly defined structure of the Neyman-Pearson theory. In particular, the latter of course permits quantitative evaluations and comparisons.

It is just this clarity and precision, at the time only an unrealized idea, that is at the heart of the debate between Bertrand and Borel. Bertrand would undoubtedly object to many of the uses to which tests are put (and he would not be alone in this). It is true, in particular, that the models from which the analysis is derived often have a rather flimsy basis and even at best are only approximations. The theory has tried to meet these and related objections by studying the robustness of existing tests, developing new more robust alternative tests, by providing simultaneous test procedures to deal with the problem of multiplicity, and so on, all within the general Neyman-Pearson framework.

To the many objections that remain, Borel has an answer in the passage quoted in Section 3 which can be summarized as follows. "The theory of probability cannot refuse to examine" the situation and to come up with an answer. Of course, the real situation is much more complex than the model allows for; the precise answers that the calculations provide for hypothetically precise questions are only rough approximate solutions of the much less precise real problems. We should therefore not take the analysis too literally, put too much stock in the precise numerical answers, but we should not give up on the enterprise. It provides invaluable guidance, a check on undisciplined speculation, on "inferences at which one arrives too quickly by 'common sense'." 


\section{REFERENCES}

Bertrand, J. (1889, 1897, 1907) Calcul des Probabilités Gauthier-Villars, Paris.

Borel, E. (1914, 1920, 1932; New Revised Edition 1948) Le Hasard. Presses Universaires de France, Paris.

Dale, A.I. (1991) A History of Inverse Probability: From Thomas Bayes to Karl Pearson, Springer Verlag, New York.

Daston, L. (1988) Classical Probability in the Enlightenment, Princeton Univ. Press.

Edgeworth, F.Y. (1895) Methods of Statistics, Jubilee Volume of the Statist. Soc., 181-217.

Fisher, R.A. (1925) Statistical Methods for Research Workers, Oliver and Boyd, Edinburgh.

Gower, B. (1982) Astronomy and probability. Forbes versus Michell on the distribution of the stars. Annals of Science, 39, 145-160.

Freedman, D. (1985) Statistics and the scientific method, Cohort Anal., 343-359.

Freedman, D. (1987) As others see us: A case study in path analysis, J. Educ. Statist., 12, 101-128.

Hochberg, Y. and Tamhane, A. (1987) Multiple Comparison Procedures, Wiley, New York.

Laplace, P.S. (1812, 1814, 1820) Théorie Analytique des Probabilités, Courcier, Paris.

Lévy, P. (1925) Calcul des Probabilités, Gauthier-Villars. 1925.

Neyman, J. (1929) Méthodes nonvelles de vérification des hypothèses. Comptes Rendus du 1 Congrès de Mathématiciens des Pays Slaves. Warsaw, 355366.

Neyman, J. (1932) On methods of testing hypotheses, Atti Congr. Internat. Matematici, 6, 35.41.

Neyman, J. (1935) Sur la vérification des hypothèses statistiques composées, Bull. Soc. Math. de France, 63, 346-366.

Neyman, J. and Pearson, E.S. (1928) On the use and interpretation of certain test criteria, Biometrika, 20A, 175-240 and 263-294.

Neyman, J. and Pearson, E.S. (1933) On the problem of the most efficient tests of statistical hypotheses, Phil. Trans. Roy. Soc. (A), 231, 289-337.

Pearson, E.S. (1939) "Student" as a statistician, Biometrika, 30, 205-250.

Pearson, E.S. (1966) The Neyman-Pearson story, 1926-34, In Research Papers in Statistics (Festschrift for J. Neyman), F.N. David (Ed). Wiley. New York.

Pearson, K. (1900) On the criterion that a given system of deviations from the probable in the case of a correlated system of variables is such that it can be reasonably supposed to have arisen from random sampling, Phil. Mag. 5, Vol. 50, 157-175.

Poisson, S.D. (1837) Recherches sur la Probabilité des Jugements, Bachelier, Paris. 
- Bertrand-Borel Debate and neyman-Pearson Theory

Reid, C. (1982) Neyman from Life, Springer-Verlag, New York.

Shaffer, J. (1988) Simultaneous testing, In Encycl. Statist. Sci. Vol. 8, 484-490. Wiley, New York.

Stigler, S. (1986) The History of Statistics, Belknap Press, Cambridge.

Student (1908) On the probable error of a mean, Biometrika, 6, 1-25.

Department of Statistics

367 Evans Hall

University of California

Berkeley, California 94720

U. S. A. 